\title{
Estimativa do padrão de preferência térmica de matrizes pesadas (frango de corte)
}

\author{
Fábio P. F. Curto ${ }^{1}$, Irenilza de A. Naas' ${ }^{1}$, Danilo F. Pereira² \& Douglas D. Salgado ${ }^{1}$
}

\begin{abstract}
RESUMO
A produtividade de matrizes pesadas (frango de corte) está ligada aos princípios de conforto térmico, diretamente relacionados ao microclima da instalação. Neste trabalho objetivou-se o monitoramento do comportamento de matrizes pesadas usando-se a tecnologia de radiofreqüência, identificadores eletrônicos injetáveis e leitores no interior de modelo de galpão de produção em escala reduzida e distorcida. Utilizaram-se no experimento oito aves com identificação eletrônica. Três antenas foram dispostas em três pontos dentro do modelo: no piso do ninho, na passagem entre a parede lateral e o comedouro e sob o bebedouro. Registraram-se continuamente as temperaturas de bulbo seco (TBS), bulbo úmido (TBU) e de globo negro (TGN). Os resultados apontam para um padrão de preferência térmica das aves diferenciado frente às situações de ambiente ocorridas no experimento. Três modelos probabilísticos de comportamento foram desenvolvidos a partir dos registros coletados: modelo probabilístico para uso da passagem: $\mathrm{FP}=1,10-0,244 \ln (\mathrm{TBS})$, modelo probabilístico para uso do bebedouro: $\mathrm{FB}=0,398$ $+0,00866$ TBS, e modelo probabilístico para uso do ninho: $\mathrm{FN}=2,22-0,272 \mathrm{TBS}+0,011 \mathrm{TBS}^{2}-0,000144 \mathrm{TBS}^{3}$.
\end{abstract}

Palavras-chave: comportamento, modelo probabilístico, identificação eletrônica

\section{Thermal preference pattern estimates of female broiler breeders}

\begin{abstract}
Female broiler breeder productivity is based on the principles of thermal comfort that are directly related with the microclimate inside the housing. This research had the objective of monitoring the behavior of female broiler breeders, using the technology of radio-frequency, injectable transponders and readers in different existing microclimates inside a small scale distorted housing model. Eight birds with electronic identification were used. Three readers were used, in three different points inside the model: on the floor of the nest, in the passage besides the lateral wall and below the water facility. Dry bulb (DBT), wet bulb (WBT) and black globe (BGT) temperature were measured continuously. The results point out a distinct behavioral pattern of the birds regarding the environment exposition during the experiment. Three probabilistic models of behavior were developed from the recorded data: probabilistic model for the passage use: FP $=1.10-0.244 \ln (\mathrm{DBT}$ ), probabilistic model for the water facility use: $\mathrm{FB}=0.398+0.00866(\mathrm{DBT})$, and probabilistic model for the nest use: $\mathrm{FN}=$ $2.22-0.272 \mathrm{DBT}+0,011 \mathrm{DBT}^{2}-0.000144 \mathrm{DBT}^{3}$.
\end{abstract}

Key words: behavior, probabilistic model, electronic identification 


\section{INTRODUÇÃO}

Os resultados produtivos de matrizes de frangos de corte podem ser avaliados em função dos efeitos climáticos no interior do galpão e do comportamento apresentado durante o período de alojamento, além de outros fatores como a genética, a nutrição e a sanidade dos animais e a própria interação entre estes, que depende diretamente do espaço ocupado. Vários autores estudaram o ambiente interno de galpões para aves desde os anos 80 (Esmay, 1982; Webster \& King, 1987; Marder \& Arad, 1989; Nääs, 1994). Também a influência do ambiente físico do alojamento na resposta produtiva de aves alojadas, assim como seu controle e acondicionamento, têm sido extensivamente descritos na literatura (Xin et al., 1994; Bottcher \& Czarick, 1997; Deaton et al., 1997; Gates et al., 1998; Hamrita et al., 1998; Hamrita \& Mitchell, 1999; Lacey et al., 2000).

Uma questão importante no tocante à produção avícola, é se alcançar condições ideais de ambiente interno no alojamento. Uma definição adequada da zona de conforto térmico pode ser indicada como sendo a faixa de temperatura ambiente em que a taxa metabólica é mínima e a homeotermia é mantida com menor gasto energético. $\mathrm{Na}$ zona de conforto térmico a fração de energia metabolizável utilizada para termogênese é mínima e a energia líquida de produção é máxima. Segundo Macari et al. (1994), no frio as aves procuram manter a homeotermia através de aumento na produção de calor e na redução de perdas; no calor, o processo é invertido. As matrizes de frango de corte apresentam grandes quantidades de músculo branco com poucas mitocôndrias, pequena vascularização e baixa capacidade calorigênica e, portanto, vulneráveis à grande variação de temperatura.

No verão, as aves procuram dissipar calor, através de mecanismos evaporativos (cutâneos e respiratórios). Respostas fisiológicas são também evidenciadas, como a queda da asa, aumento de área superficial (crista e barbela), erição das penas (arrepiar) e outras. As aves podem aumentar drasticamente a freqüência respiratória e ventilar, de forma eficaz, as vias aéreas superiores. A capacidade das aves de dissipar calor diminui a medida em que a temperatura ambiente e a umidade relativa se elevam acima da zona termoneutra; como resultado, a temperatura corporal da ave sobe e logo aparecem os sintomas do estresse calórico. A redução de consumo alimentar diminui os substratos metabólicos ou combustíveis disponíveis para o metabolismo reduzindo, desta forma, a produção de calor (Teeter \& Belay, 1993). Rutz (1994) observou que quando o ambiente térmico se encontra acima da condição de termoneutralidade das aves, suas atividades físicas são reduzidas a fim de diminuir a produção interna de calor. Devido à vasodilatação e ao acréscimo da circulação periférica, suas cristas e barbelas aumentam de tamanho e se tornam mais avermelhadas, contribuindo para a perda de calor sensível, enquanto o ofego se torna eficiente apenas quando a umidade relativa do ar se encontra em níveis relativamente baixos (Lasiewski et al., 1966). Nesta ocasião há, geralmente, recusa natural à alimentação e a prostração é evidenciada. As conseqüências mais importantes do excesso de calor são: queda do consumo de alimentos, aumento do consumo de água, menor taxa de crescimento, queda na pro- dução de ovos, maior incidência de ovos com casca mole e de menor densidade, diminuição da eclodibilidade, além de aumento da mortalidade.

Segundo Kebeler \& Schiefer (1998), as tarefas que compõem as atividades agrícolas e são executadas em um sistema de produção, necessitam de acompanhamento sistemático. Os avanços tecnológicos na área de informática, juntamente com a utilização de instrumentos desenvolvidos dentro da área de instrumentação agrícola utilizados com softwares aplicativos, conforme Zambalde (1996), são capazes de proporcionar, ao empresário da área rural, ferramentas poderosas para melhor gerenciamento dos seus negócios.

Korthals et al. (1992) relataram o uso de transponder, que é a combinação das palavras em inglês: transmitter e responder e que recebe e transmite um sinal de rádio a certa freqüência, através de um microchip tendo em cada elemento um código numérico único para o monitoramento de respostas bioenergéticas; quando utilizaram o monitoramento intensivo de consumo de alimentos, produção de calor e comportamento dos animais, por meio de equipamentos especiais, como: identificação individual dos animais, comedouros automáticos com pesagem, calorímetros, registro de imagens e registradores de temperatura estacionários e portáteis, baseados em microprocessadores. Em pesquisa realizada por Hamrita \& Mitchell (1999), investigou-se o uso de um sistema de biotelemetria para medição da temperatura interna de aves e os resultados indicam que equipamentos de telemetria são adequados para o monitoramento contínuo da temperatura interna corporal das aves desta variável.

O efeito da variável ambiente sobre um sistema de produção pode ser medido e ajustado se os índices desejáveis forem conhecidos. A precisão com que esses índices são alcançados está relacionada à capacidade de interferência nos processos e com o tempo necessário para que os ajustes possam ser realizados. Godfrey (1986) apresentou um estudo relacionado aos parâmetros de modelos usados em biomedicina, em que a questão principal foi a identificação desses parâmetros. Para modelos lineares existem diversas aproximações, porém o autor considerou que apenas uma das aproximações (baseada na expansão da série de Taylor) das observações deste estudo foi diretamente aplicável para sistemas não lineares. Em estudo realizado por Altmann \& Altmann (1977), os autores demonstraram que modelos probabilísticos são eficientes para estimar a expectativa de freqüência de comportamento e de interação social em cada categoria de idade e gênero, particularmente quando o estudo de população contém mudança dinâmica.

Neste cenário objetivou-se através do presente trabalho, interpretar a preferência térmica de matrizes pesadas (frangos de corte), utilizando-se do sistema de monitoramento individual eletrônico e definir um modelo probabilístico para cálculo do valor esperado de frequência de ocorrência dos comportamentos em função das variáveis do ambiente de alojamento.

\section{MATERIAL E MÉTODOS}

O experimento foi conduzido no Laboratório de Conforto Ambiental da Faculdade de Engenharia Agrícola da 
UNICAMP, situado a latitude de $22^{\circ} 54^{\prime} \mathrm{S}$, longitude de $47^{\circ}$ $05^{\prime} \mathrm{N}$ e altitude de $674 \mathrm{~m}$, tipo climático Cwa, segundo a classificação de Köeppen, caracterizado como clima tropical de altitude, com inverno seco e verão quente, localizado no município de Campinas, SP, em dois protótipos de modelos em escala reduzida e distorcida de parte de galpões de produção. Os modelos em escala reduzida e distorcida foram construídos na direção Leste-Oeste, sendo o seu entorno composto de área plana coberta de grama. As escalas utilizadas são 1:10, nas dimensões horizontais, e 1:2 nas dimensões verticais. Apresentam-se na Tabela 1, as dimensões do modelo. O modelo era aberto possuia as laterais norte e sul abertas, e tinha uma de mureta de $15 \mathrm{~cm}$ construído de $1 / 2$ tijolo assentado com argamassa de cimento e areia, para fixação da tela. $\mathrm{O}$ piso em concreto foi recoberto com $10 \mathrm{~cm}$ de maravalha, e o telhado em duas águas com telhas de fibrocimento e orientação Leste-Oeste, com dimensões de 1,40 x 3,0 x 1,5 m.

Tabela 1. Dimensões do modelo em escala natural e distorcido

\begin{tabular}{lccc}
\hline \multicolumn{1}{c}{ Escala } & $\mathbf{1 : 1}$ (natural) & $\mathbf{1 : 1 0}$ (horizontal) & $\mathbf{1 : 2}$ (vertical) \\
Largura $(\mathrm{m})$ & 14,00 & 1,40 & - \\
Comprimento $(\mathrm{m})$ & 30,00 & 3,00 & - \\
Pé direito $(\mathrm{m})$ & 3,00 & - & 1,50 \\
Altura mureta $(\mathrm{m})$ & 0,20 & - & 0,10 \\
\hline
\end{tabular}

Para registro dos dados do ambiente usaram-se três termopares tipo J, conectados através de cabos a uma altura de $1 \mathrm{~m}$ da cama de maravalha, no centro dos galpões, com a finalidade de coletar as informações de Temperatura de Bulbo Seco (TBS), Temperatura de Bulbo Úmido (TBU) e Temperatura de Globo Negro (TGN).

A técnica de identificação das aves utilizada baseou-se no uso de dispositivos eletrônicos para detectar o código ou número de identificação contido em um transponder. Foi utilizada a tecnologia de rádio freqüência da TROVAN ${ }^{\circledR}$, com método de duplicidade FDX, freqüência de ativação de $128 \mathrm{kHz}$, modulação PSK, freqüência de entorno $64 \mathrm{kHz}$, codificação DPB, paridade $8 \mathrm{kbit} \mathrm{s}^{-1}$, que consiste na comunicação entre um transponder e um leitor. O leitor (antena) possuía a dimensão de 40,5 x 40,5 cm, com espessura de $2,5 \mathrm{~cm}$, tendo um controlador que transforma o sinal analógico no código no padrão ASCII e disponibiliza transmite a informação para registro no computador, por meio de cabo serial padrão RS232, registrando a leitura do código do transponder.

Os leitores foram estrategicamente colocados no ninho, embaixo do bebedouro, e entre o comedouro (posicionado no centro do galpão faceando a parede sul) e a parede norte, que foi denominado como a passagem entre as paredes laterais. Todas as antenas foram dispostas sob embaixo da cama de maravalha de maneira a registrar a movimentação das aves ao se deslocarem entre o bebedouro e o comedouro de um lado para o outro do galpão (leste-oeste) na antena da passagem e as frequências de uso do bebedouro e do ninho, sem alterar o seu acesso usual à cama e, desta maneira, eliminar eventuais desvios de comportamento), e no ninho. Os dados de frequência de uso dos locais foram registrados por um através de uma conexão com um software, fazer o monitoramento da movimentação das matrizes através da captação da informação contida nos transponders e a correspondência ao evento de identificação da ave desenvolvido em MS-Visual Basic 6.0, que correlacionou os dados de comportamento com os de ambiente e permitiu o monitoramento eletrônico das aves e o gerenciamento eficiente dos registros.

Os trabalhos foram conduzidos com 8 (oito) matrizes de frango de corte da linhagem Hybro-G e utilizadas no experimento, oito aves com 28 semanas de idade, que tiveram os transponders implantados subcutaneamente na perna, utilizando-se as seringa e agulha próprias.

Como a antena comercial já possuía a característica de acionamento automático para a leitura do código do transponder, foi possível fazer, por meio da configuração do sistema, a sua leitura a cada $2 \mathrm{~s}$, sendo que em um mesmo transponder poderia ser lido somente duas vezes a cada $60 \mathrm{~s}$.

As temperaturas limites foram estabelecidas segundo a literatura (Esmay, 1982; Webster \& King, 1987; Marder \& Arad, 1989), sendo TCA, temperatura crítica alta $=32{ }^{\circ} \mathrm{C}$ e TCB, temperatura crítica baixa $=20{ }^{\circ} \mathrm{C}$. Estabeleceram-se duas situações: dentro da Zona de Termoneutralidade (ZTN) e fora da ZTN.

Os estados de comportamento das aves no interior dos modelos identificados foram: 1) momentos em que se encontravam no ninho; 2) ida ao bebedouro para hidratação e 3) atividade motora dentro do galpão (utilizando a passagem)

$\mathrm{O}$ experimento foi conduzido de forma a registrar continuamente as informações referentes ao estado de comportamento em que as aves se encontravam em períodos de $24 \mathrm{~h}$, de outubro de 2000 a fevereiro de 2001. Realizaram-se coletas de informações referentes ao dia e ao horário de coleta, ao mesmo tempo em que ocorriam valores de temperatura de bulbo seco (TBS), temperatura de bulbo úmido (TBU) e temperatura de globo negro (TGN).

Para a análise estatística, utilizaram-se todos os dados registrados no período experimental. O programa computacional Minitab ${ }^{\circledR}$ foi usado para a análise estatística e na análise de regressão, para descrever a freqüência de utilização dos locais monitorados, em função da TBS e UR.

\section{RESULTADOS E DISCUSSÃO}

Durante o período experimental, foram coletados aproximadamente 520.000 registros da posição das aves no interior do modelo. No nível de significância de $5 \%$, não se encontrou evidência para se rejeitar a hipótese de homogeneidade dos dados; considerou-se, portanto, que as freqüências relativas foram estatisticamente homogêneas.

Para determinar a influência da ZTN no comportamento das aves em função da freqüência de utilização dos locais de posicionamento das antenas, realizou-se o teste de Qui-quadrado para verificação da existência de diferença entre o número de leituras nos locais de posicionamento das antenas, independente do fato das aves estarem ou não sob condição de ZTN. Os resultados provenientes da análise estatística realizada com todos os dados do período de testes demons- 
traram que houve influência significativa da alteração do comportamento, em função da ZTN; entretanto, durante este período de coleta de dados a incidência de dados climáticos fora da ZTN foi muito maior que dos dados dentro da ZTN, por se tratar de um período quente do ano. O P-Valor $=0,00$ indicou que a associação de movimentação das aves (em ZTN e fora de ZTN) e locais de posicionamento das antenas foi significativa a qualquer nível de significância; em seguida, realizou-se uma análise para verificar cada variável que influencia o comportamento das aves, mostrando a alteração de seu comportamento, de acordo com valores de TBS, em termos

Tabela 2. Classes de valores de temperatura (TBS) e umidade relativa (UR)

\begin{tabular}{ccc}
\hline Classes & TBS $\left({ }^{\circ} \mathbf{C}\right)$ (Amplitude) & UR (\%) (Amplitude) \\
1 & $14,80-17,60$ & $40,00-47,50$ \\
2 & $17,61-20,40$ & $47,51-55,00$ \\
3 & $20,41-23,20$ & $55,01-62,50$ \\
4 & $23,21-26,00$ & $62,51-70,00$ \\
5 & $26,01-28,80$ & $70,01-77,50$ \\
6 & $28,81-31,60$ & $77,51-85,00$ \\
7 & $31,61-34,40$ & $85,01-92,50$ \\
8 & $34,41-37,20$ & $92,51-100,00$ \\
\hline
\end{tabular}

de freqüência de utilização de determinados locais. Para isto, fez-se oportuna a definição de uma amplitude por classes de valores de temperatura e umidade relativa, selecionada dentro do universo de dados ocorridos no período experimental, conforme indica a Tabela 2 .

Os resultados indicados na Tabela 3 mostram as diferenças nas freqüências de utilização do ninho, bebedouro e da passagem, em função das classes de TBS, demonstrando haver alteração no comportamento, em função dos valores de TBS, também citado por Macari et al. (1994); os autores explicam que no verão as aves procuram dissipar calor através de mecanismos evaporativos (cutâneos e respiratórios) e o demonstram através de mudanças no padrão comportamental.

Verificou-se queda na freqüência de registros na passagem, quando ocorre um acréscimo na TBS (Classes 5 a 8), e tendência de acréscimo na freqüência de registros no bebedouro, também em virtude do acréscimo de TBS. Esta observação pode ser justificada através dos resultados apresentados

Tabela 3. Freqüência de utilização de cada local em função da temperatura ambiente e da umidade relativa do ar, para cada classe de cada fator ambiental

\begin{tabular}{|c|c|c|c|c|c|c|c|}
\hline \multirow{3}{*}{ Classes } & \multicolumn{6}{|c|}{ Freqüência de utilização (\%) } & \multirow{3}{*}{ Tota } \\
\hline & \multicolumn{2}{|c|}{ Bebedouro } & \multicolumn{2}{|c|}{ Passagem } & \multicolumn{2}{|c|}{ Ninho } & \\
\hline & TBS & UR & TBS & UR & TBS & UR & \\
\hline 1 & 52 & 76 & 42 & 21 & 6 & 2 & 100 \\
\hline 2 & 57 & 70 & 40 & 25 & 3 & 5 & 100 \\
\hline 3 & 59 & 64 & 37 & 28 & 4 & 9 & 100 \\
\hline 4 & 59 & 59 & 31 & 32 & 9 & 10 & 100 \\
\hline 5 & 61 & 63 & 30 & 30 & 10 & 7 & 100 \\
\hline 6 & 70 & 63 & 24 & 30 & 7 & 7 & 100 \\
\hline 7 & 72 & 57 & 23 & 37 & 5 & 6 & 100 \\
\hline 8 & 67 & 57 & 27 & 39 & 6 & 4 & 100 \\
\hline
\end{tabular}

por Freeman (1988) que evidenciou o efeito das variáveis de ambiente no interior do galpão e no consumo de água pelas aves. Os resultados observados coincidem também com os dados de Macari et al. (1994) quando evidenciaram que as aves regulam a produção de calor e a redução de perdas, em função de exposição a temperaturas altas ou baixas.

Para avaliação da alteração do comportamento das aves, de acordo com os valores de UR foi imprescindível a definição de uma amplitude por classes de valores de umidade relativa, conforme Tabela 2.

Realizou-se o teste de Qui-quadrado para se verificar se houve diferença no comportamento das aves devido às diferentes classes de UR. Os resultados mostraram haver associação, a qualquer nível de significância $(\mathrm{P}-$ Valor $=0,00)$, entre as classes de UR e o uso do ninho, do bebedouro e da passagem, fato que demonstra haver alteração no comportamento, em função dos valores de UR. Esta observação justifica, também através dos resultados obtidos por Teeter \& Belay (1993) quando foi demonstrado em câmara climática com distintas combinações de TBS e UR, que as aves reduzem o consumo de alimentos quando a temperatura se eleva acima da ZTN, concomitantemente com a elevação da umidade relativa do ar, sendo que esta redução diminui os substratos metabólicos ou combustíveis disponíveis para o metabolismo e, em contrapartida, reduzem a produção de calor. O comportamento observado se justifica também através das evidências apresentadas por Rutz (1994) no tocante à redução da atividade física de aves expostas ao estresse térmico.

A Tabela 4 mostra ainda as freqüências de utilização do ninho, bebedouro e passagem, devido às distintas classes de UR. Observa-se tendência de acréscimo na freqüência de registros na passagem, quando há acréscimo na UR, enquanto ocorre tendência de diminuição na freqüência de registros no bebedouro, em virtude do acréscimo de UR, concordando com o encontrado por Lasiewski et al. (1966).

Tabela 4. Freqüência de utilização de cada local em função da umidade relativa do ar, para cada classe

\begin{tabular}{cccccc}
\hline \multirow{2}{*}{ Classes } & \multirow{2}{*}{ UR (\%) } & \multicolumn{4}{c}{ Freqüência de utilização (\%) } \\
\cline { 3 - 6 } & & Bebedouro & Passagem & Ninho & Total \\
\hline 1 & $40,0-47,5$ & 76 & 21 & 2 & 100 \\
2 & $47,5-55,0$ & 70 & 25 & 5 & 100 \\
3 & $55,0-62,5$ & 64 & 28 & 9 & 100 \\
4 & $62,5-70,0$ & 59 & 32 & 10 & 100 \\
5 & $70,0-77,5$ & 63 & 30 & 7 & 100 \\
6 & $77,5-85,0$ & 63 & 30 & 7 & 100 \\
7 & $85,0-92,5$ & 57 & 37 & 6 & 100 \\
8 & $92,5-100,0$ & 57 & 39 & 4 & 100 \\
\hline
\end{tabular}

Foram estudadas, então, as possibilidades de geração de um modelo probabilístico relacionado ao comportamento das matrizes de frango de corte, nas condições ambientais registradas e, para freqüência de utilização da passagem, encontrou-se o seguinte modelo:

$$
\mathrm{FP}=1,10-0,244 \ln (\mathrm{TBS})
$$


em que:

$\mathrm{FP}$ - freqüência da passagem, para $\mathrm{a}=1 \%$, com $\mathrm{R}^{2}=0,804$

Por meio da ANOVA encontrou-se a significância do modelo (Eq. 1), com P-Valor $(0,00)$ do teste F e P-Valor $(0,00)$ do teste $\mathrm{T}$, verificando-se a significância de cada variável no modelo de regressão. Este modelo teve sustentação no trabalho de Altmann \& Altmann (1977) quando ficou demonstrado que equações podem ser desenvolvidas para o cálculo da expectativa de freqüência de comportamento.

A Figura 1 ilustra a freqüência de utilização da passagem pelas aves, em função da TBS, mostrando que a passagem foi utilizada de maneira inversamente proporcional ao acréscimo de TBS, indicando que houve menor movimentação das aves por ocasião do aumento da temperatura ambiente. Macari et al. (1994), Rutz (1994), Nääs (1994) apontam a situação de prostração frente a altos valores de TBS, o que reforça o perfil da curva encontrada.

Para a freqüência de utilização do bebedouro, encontrouse o seguinte modelo:

$$
\mathrm{FB}=0,398+0,00866(\mathrm{TBS})
$$

em que:

FB - freqüência do bebedouro, para $\mathrm{a}=1 \%, \mathrm{com}^{2}=0,66$

Encontrou-se através da ANOVA, a significância do modelo (Eq. 2), com P-valor $(0,00)$ do teste F e P-valor $(0,00)$ do teste $\mathrm{T}$, verificando-se a significância de cada variável no modelo de regressão. A Figura 1 ilustra ainda a freqüência de utilização do bebedouro em função da TBS, indicando que este local foi utilizado de maneira diretamente proporcional ao acréscimo de TBS, e que houve maior número de visitas das aves ao bebedouro, quando ocorreu o aumento da temperatura ambiente. Macari et al. (1994) e Teteer \& Belay (1993) observaram que havia um acréscimo no consumo de água

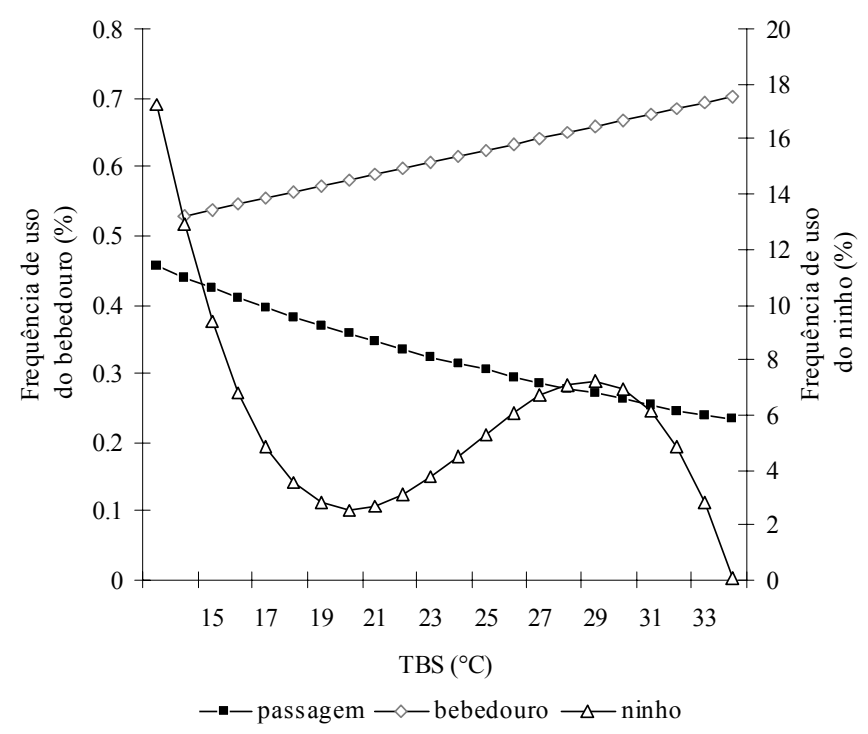

Figura 1. Freqüência de utilização da passagem, do bebedouro e do ninho, em função da temperatura ambiente quando da ocorrência de altos valores de TBS, coincidente com o que descreve o modelo deste experimento.

Para a freqüência de utilização do ninho encontrou-se o seguinte modelo:

$\mathrm{FN}=2,22-0,272 \mathrm{TBS}+0,011 \mathrm{TBS}^{2}-0,000144 \mathrm{TBS}^{3}$

em que:

$\mathrm{FN}$ - freqüência do ninho, para $\mathrm{a}=5 \%, \operatorname{com~}^{2}=0,421$.

Encontrou-se, através da ANOVA, a significância do modelo (Eq. 3) com P-Valor $(0,01)$ do teste F e P-Valor $(0,018)$ do teste $\mathrm{T}$, verificando-se a significância de cada variável no modelo de regressão. A freqüência de utilização do ninho, em função da TBS, pode ser visto na Figura 1, comprovando que o ninho foi utilizado da seguinte maneira: para temperatura baixa $\left(<20^{\circ} \mathrm{C}\right)$ notou-se queda da freqüência de utilização do ninho (vide número de pontos indicando a presença de ave no ninho) ocorrendo daí um número pequeno de leituras; para temperaturas entre 20 e $30^{\circ} \mathrm{C}$, a função mostra uma utilização constante e, para temperaturas superiores acima de $30{ }^{\circ} \mathrm{C}$, fica evidenciado o decréscimo de utilização do ninho (vide o número de pontos registrados).

\section{CONCLUSÕES}

1. Para o presente estudo, o sistema de monitoramento remoto permitiu conhecer o comportamento de preferência térmica das aves, em função da exposição ao ambiente, sem a interferência humana sendo possível quantificar o uso de bebedouro, passagem e ninho, durante o período analisado.

2. Ficou evidente que as aves utilizaram, com maior freqüência, o bebedouro com o acréscimo da temperatura ambiente.

3. Verificou-se, ainda, menor freqüência de utilização da passagem demonstrando menor atividade motora das aves, quando ocorreu acréscimo da temperatura ambiente.

4. A freqüência de utilização do ninho foi maior quando a temperatura ambiente esteve próxima da ZTN, enquanto diminuiu na medida em que se deu acréscimo da temperatura ambiente.

\section{LITERATURA CITADA}

Altmann, S.A.; Altmann, J. On the analysis of rates of behaviour. Animal Behaviour, Londres, v.25, n.1, p.364-372, 1977.

Bottcher, R.W.; Czarick, M. Tunnel ventilation and evaporative cooling for poultry. North Carolina: Cooperative Extension Service, AG-554, 1997.11p.

Deaton, J.W.; Reece, F.N.; McNaughton N. The effect of temperature during the growing period on broiler performance. Poultry Science, Savoy, v.57, n.1, p.1070 - 1074, 1997.

Esmay, M.L. Principles of animal environment. Westport: AVI Publication Coorporation Inc., 1982. 325p. 
Freeman, B.J. The domestic fowl in biomedical research: physiological effects of the environment. World's Poultry Science Journal, Londres, v.44, n.2, p.44-60, 1988.

Gates, R.S.; Zhang, H.; Colliver, D.G.; Overhults, D.G. Regional variation in temperature humidity index for poultry housing. Transactions of the ASAE, Saint Joseph, v.38, n.1, p.197205, 1998.

Godfrey, K.R. The identifiably of parameters of models used in biomedicine. Mathematical Modeling, Newport, v.7, n.1, p.1195-1214, 1986.

Hamrita, T.K.; Mitchell, B. Poultry housing control: A summary of where we are and where we want to go. Transactions of the ASAE, Saint Joseph, v.42, n.2, p.479-483, 1999.

Hamrita, T. K.; van Wicklen, G.; Czarick, M.; Lacy, M. Monitoring poultry deep body temperature using biotelemetry. Applied Engineering in Agriculture, Saint Joseph, v.14, n.3, p.327-331, 1998.

Kebeler, T.; Schiefer, G. Computer aided environmental control in environmental-management-systems in agricultural and food industrial production chains and its tools. In: International Conference on Sustainable Agriculture for Food, Energy and Industry, 1998, Braunschweig. Anais... Braunschweig: El Bassam N, Behl RK, Prochnow B, 1998, v.2, p.700-706.

Korthals, R.L.; McDonald, T.P.; Eigenberg, R. A. Experiences with transponders for monitoring bioenergetic responses. In: ASAE Meeting, 23, 1992, Charlotte. Anais... Saint Joseph, MI: ASAE, 1992 p.8.

Lacey, B.; Hamrita, T.K.; Mitchell, B. Feasibility of using neural networks for real-time prediction of poultry deep body temperature responses to stressful changes in ambient temperature. Applied Engineering in Agriculture, Saint Joseph, v.16, n.3, p.303-308, 2000.
Lasiewski, A.; Acosta, L.; Bernstein, M.H. Evaporative water loss in birds I. Characteristics of the open flow method of determination, and their relation to estimates of thermoregulatory ability. Comparative Biochemistry and Physiology, Montreal, v.19, n.1, p.445-457, 1966.

Macari, M.; Furlan, R.L.; Gonzales, E. Fisiologia aviária aplicada a frangos de corte. Jaboticabal: FUNEP/UNESP, 1994. 296p.

Marder, J.; Arad Z. Panting and acid-base regulation in heat stressed birds. Comparative Biochemistry and Physiology, Montreal, v.94, n.A, p.395-400, 1989.

Nääs, I.A. Aspectos físicos da construção no controle térmico do ambiente das instalações. In: Conferência APINCO, Ciência e Tecnologia Avícola, 1994, Santos. Anais...Santos: FACTA, 1994, p.158-167.

Rutz, F. Aspectos fisiológicos que regulam o conforto térmico as aves. In: Conferência APINCO, Ciência e Tecnologia Avícola, 1994, Santos. Anais...Santos:FACTA, 1994, p.99110.

Teeter, R.G., Belay, T. Broiler water balance and thermobalance during thermoneutral and high ambient temperature exposure. Poultry Science, Savoy, v.72, p.116-124, 1993.

Webster, M.D.; King, J.R. Temperature and humidity dynamics of cutaneous and respiratory evaporation in pigeons. Journal of Comparative Physiology, Toronto, v.87, n.2, p.157253, 1987.

Xin, H.; Berry, I.L. Tabler, G.T. Barton, T.L. Temperature and humidity profiles of broiler houses with experimental conventional and tunnel ventilation. Transactions of the ASAE, Saint Joseph, v.10, n.4, p.535-542, 1994.

Zambalde, A.L. Considerações estratégicas sobre o processo de informatização das empresas e propriedades rurais. Revista Agrosoft, Lavras, v.1, n.1, p.1-7, 1996. 